\title{
What is the role of the distraction test of hearing?
}

\author{
Alison Mott, Alan Emond
}

\begin{abstract}
The use of the distraction test as a screen for hearing loss in infancy was reviewed in the Bristol and District Health Authority. Inaccuracies in data recording were found which challenged the screening and referral rates produced by the National Child Health Computer. Representative samples of children failing the distraction test and children needing hearing aids were followed up through the screening process. Of 130 children failing the test, only $46(35 \%)$ had moderate conductive hearing loss and none had a severe loss. Of 34 children with sensorineural hearing loss (SNHL) requiring hearing aids, seven $(21 \%)$ were identified by the test and five $(15 \%)$ presented late after having been screened.

The distraction test as now used in Bristol identifies large numbers of children with minor hearing loss. The referral of these children to the secondary services leads to delays for those children with more significant loss. The test is no longer the main method for identifying children with SNHL.
\end{abstract}

(Arch Dis Child 1994; 70: 10-13)

Early detection of hearing problems is considered essential to ensure appropriate management during a critical stage of language development. The prevalence of hearing loss requiring treatment in childhood is estimated at $0.15 \%$ for sensorineural hearing loss (SNHL) and 3\% for conductive hearing loss due to otitis media with effusion. ${ }^{1}$ The Hall report $^{2}$ recommends the routine testing of hearing in infancy as part of a programme of child health surveillance.

Most health districts use the distraction test to assess hearing from the age of 7 months. The distraction test was originally introduced to detect SNHL and has been proved to be effective. ${ }^{34}$ The continuing use of the distraction test has been justified on the grounds that $30 \%$ of infants with SNHL have no tisk factors, ${ }^{5}$ and parental awareness of hearing loss may be unreliable. ${ }^{6}$ Concerns have been expressed about the large numbers of children with otitis media with effusion, rather than SNHL, who fail the distraction test and are referred to the audiological services. Studies have questioned the distraction test's objectivity and repeatability ${ }^{7-9}$ and the significance and treatment of otitis media with effusion remains controversial. ${ }^{1011}$ As a result, some health authorities have abandoned the test as a routine screening measure. ${ }^{12}$

In Bristol, there is a coordinated audiological service covering four trusts with a standardised protocol and unified training across the trusts. The service utilises the National Child Health Computer system to send appointments for screening and record the outcome of the tests. A distraction test is offered to all children at 7 months of age by their health visitor. Two failures of the test lead to referral to a community audiological clinic for assessment. If concerns persist, children are referred to the hearing centre at the Royal Hospital for Sick Children, Bristol.

We report a study of the distraction test with the aim of establishing the screening and referral rates and reviewing the accuracy of the data on the computer. The second part of the study assessed the outcome of referrals from the distraction test. The final part of the study reviewed the notes of children wearing hearing aids with severe hearing loss to identify the role of the distraction test in their diagnosis.

\section{Methods}

Using a total population sample of children resident in Bristol and district in 1990, the numbers of children screened and referred from the distraction test were obtained from the National Child Health Computer.

To check the accuracy of the computer data, two samples were selected. The first was a random sample of the notes of 100 children who had been identified as 'non-screened' by the computer. The second was a cohort of children who had failed the distraction test and were identified through the district hearing centre. This cohort was chosen using two criteria: that the children were born in 1986 and were resident in the Bristol and Weston Health Authority in 1990. Children born in 1986 were chosen as this enabled missed cases of SNHL identified through hearing aid prescription to be included. The total number of children from this cohort who failed the distraction test was 688 , and a 1:4 sample was selected. For the two samples the computer data were compared with the results recorded in the child's notes.

The second cohort was used for the next stage of research in which the children were followed through the audiological referral process. Forty two of the 172 children in the sample were subsequently excluded because they did not fit the original cohort criteria. The records of the remaining 130 children were studied for the results of hearing assessment, subsequent management, and outcome.

We classified hearing loss in the audiology clinic as mild, moderate, or severe according to the special needs module codes for the National Child Health system. Mild loss was a unilateral or 20-40 dBA loss in the better ear, moderate loss was 41-70 dBA loss, and severe loss was greater than $70 \mathrm{dBA}$ loss in the better ear. 


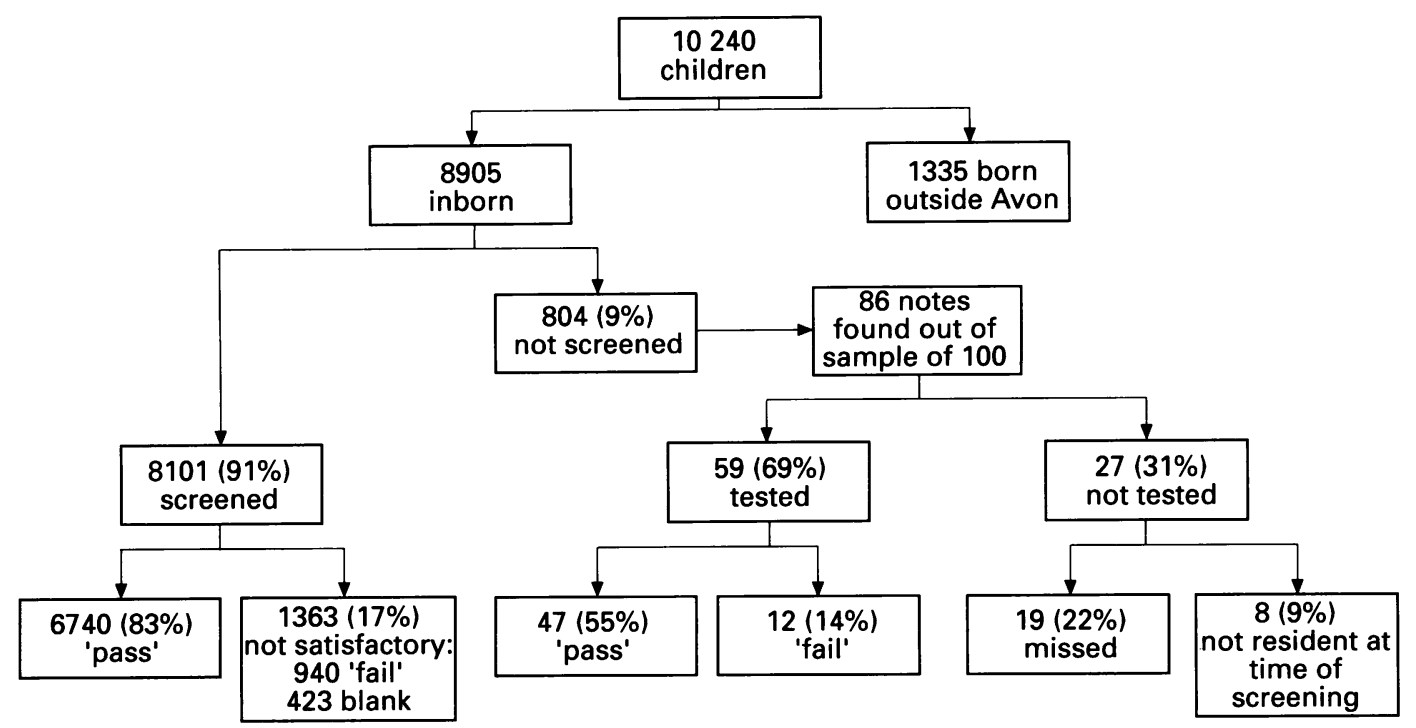

Results of distraction testing for children born in 1986.

Finally, we reviewed the notes of all children born in 1986-8 who had been prescribed hearing aids at the hearing centre and assessed the contribution of the distraction test in their diagnosis.

\section{Results}

SCREENING AND REFERRAL RATES

According to the National Child Health Computer, the screening rate was $91 \%$, the pass rate was $83 \%$, and the referral rate was $17 \%$ (see figure). Of the sample of 100 'unscreened children' the notes of 86 were found and reviewed. Fifty nine $(69 \%)$ had in fact been screened and eight $(9 \%)$ had already been referred or were no longer living in Bristol. Only 19 (22\%) were 'true' nonscreened and vulnerable to undiagnosed hearing loss. Of the 130 children known to have failed the distraction test the computer recorded $84(65 \%)$ as failing the test, $23(18 \%)$ as having evidence of the test but no clear record of the result, $10(8 \%)$ as having passed the test, and $13(10 \%)$ as non-screened.

The quality of the data on the computer makes it difficult to determine the exact referral rate. Furthermore, the coding of referrals in 1986 was imprecise as non-satisfactory codes included those returned as blank or 'observe'. An estimated referral rate was between 11.6 and $16.8 \%$ (940 and 1363 out of 8101$)$.

\section{OUTCOME OF REFERRALS FROM THE \\ DISTRACTION TEST}

The results of the hearing assessments in the community audiology clinic of the cohort of referred children showed that 44 (34\%) had normal hearing and normal tympanometry, 10 (8\%) had normal hearing and abnormal tympanometry, $24(18 \%)$ had mild hearing loss, and 46 (35\%) moderate hearing loss. No child had severe hearing loss or sensorineural hearing loss, and six children did not attend the clinic.

The table gives the final outcomes of referral. The severity of hearing loss at the initial assessment was compared with the outcome of referral to the ear, nose, and throat clinic. Thirty five $(76 \%)$ children with moderate hearing loss were referred with $23(50 \%)$ having an operation, whereas only eight (35\%) children with mild hearing loss were referred with five $(22 \%)$ having an operation.

The mean age of referral by the health visitor was 8 months (range 6-13); however, the mean age at the operation was 25 months (range 11-53). The waiting times for first appointment at the community audiology clinic was $2 \cdot 1$ months (range $0 \cdot 7-7 \cdot 7$ ), for the ear, nose, and throat clinic six months (range $0-8 \cdot 6$ ), and for an operation $4 \cdot 7$ months $(0 \cdot 5-12 \cdot 8)$. Although the waiting times for the initial assessment at community audiology clinics were short, children were often reviewed several times before referral to the ear, nose, and throat clinic. The mean waiting time for an appointment at the ear, nose, and throat clinic for children with moderate hearing loss was 4.9 months and for those with mild loss $5 \cdot 8$ months.

\section{HEARING AID WEARERS}

Of a total of 30000 births in 1986-8, 63 children were known to be wearing hearing aids; the notes of 61 children were found. Thirty four (56\%) children had pure SNHL, $18(30 \%)$ had a conductive hearing loss of whom $12(67 \%)$ had Down's syndrome, and nine $(15 \%)$ children had a mixed hearing loss. The estimated prevalence of SNHL from these data is $1 \cdot 4 / 1000$, a figure similar to many other studies. Fifty one $(84 \%)$ children had had the distraction test; of the $10(16 \%)$ children who had not, seven had already been diagnosed as

Final outcomes of referral to audiological services by health visitors $(n=130)$. Values are No $(\%)$ of children referred

Operation

No operation, still under review

Discharged at first visit

Discharged after

Discharged after repeated default $30(23)$ 4 (3) 4 (3)
45 (35) 45 (35)
34 (26) 6 (5) $11(8)$ 
having a hearing problem and three were referred early to the audiological services because of Down's syndrome.

The distraction test identified seven of the $34(21 \%)$ children with SNHL. There was no risk factor for $14(41 \%)$ children with SNHL, and half of these were identified by the distraction test. Five children born in 1986-7 with SNHL presented at over 18 months of age, four children having passed the test and one child failing the distraction test but passing the secondary assessment. It is not known if these children are true false negatives or had developed a progressive hearing loss.

The median age at which a hearing aid was fitted decreased over the three years from 28 months to 11.4 months for those children with SNHL. The proportion referred by neonatologists increased during the same period from 33 to $77 \%$.

\section{Discussion}

This study reviews the use of the distraction test to screen for hearing loss in infancy in Bristol. In the process of the study it was difficult to determine the exact referral rate from the computer as some distraction test result forms were returned as blank. In addition, some children in the samples may yet present with hearing problems. However, we are able to identify particular aspects of the use of the distraction test which need review.

The National Child Health Computer is the central source of information for overall coverage and referral rates for screening and for contacting individual children identified as 'non-screened'. The computer data in this study were shown to be inaccurate, however, with some children who had been screened recorded as non-screened and children who had failed the distraction test recorded as passing the test. It is important that health visitors, general practitioners, and clinical medical officers complete computer returns accurately and understand the significance of the codes. Resources will be wasted chasing up children who appear to be non-screened when, in fact, their hearing status is known. The introduction of records held by parents has provided an opportunity to simplify the codes (satisfactory, referred, or treatment being received) and modify the computer return forms. Regular audit of the information on the computer is needed and has now been implemented on a two yearly cycle in the Bristol and District Health Authority.

This study shows that the distraction test in Bristol is mainly identifying children with conductive hearing loss. Large numbers of children are referred and put great strain on the audiological services. The results of the secondary assessment show that although a large number of children with hearing problems are correctly identified, a third of children referred had normal hearing. This may reflect a poor screening technique or poor sensitivity of the test. With all testers being trained and regularly updated by the same team of audiologists, we believe that the screening technique is not the problem. Otitis media with effusion is a fluctuating disorder and therefore it is difficult to determine the false positive rate and the sensitivity and specificity cannot be calculated. This shows the problems of using a screening test in detecting a disorder which does not fit screening criteria. ${ }^{1314}$ Referring a common fluctuating disorder results in an overloading of the secondary system and long waiting times for children with significant hearing loss who need further treatment.

We also show that the distraction test is no longer the main screening method in the identification of children with SNHL. Although most children with SNHL wearing hearing aids had had the distraction test, only $20 \%$ were identified from the test. In fact, some children passed the test. The distraction test may still have a role as it identified half the children with no risk factors. Increasing the awareness of parents and professionals through the use of checklists may further limit the usefulness of the distraction test. In addition, riskbased neonatal screening is leading to earlier detection of children with SNHL.

One possible solution would be to abandon the test as a screen and to use it only when there are concerns about a child's hearing. This approach is being evaluated in some districts and the results are awaited with interest. Alternatively, the present system could stratify children failing the distraction test by severity of hearing loss. A secondary assessment by audiological technicians would confirm persistent and severe cases, which would be seen by a combined audiology/ear, nose, and throat clinic more quickly. The milder cases would be offered reassurance and simple advice on language stimulation and the need for visual cues. The primary health care team would be involved in subsequent follow up, and instructed on the criteria for re-referral to secondary audiology services. The expanded use of technicians to provide the initial assessment is being piloted in Bristol and will be evaluated in future audit cycles.

Further research is required into the costs and benefits of switching from a universal distraction test to a more selective policy. This study shows the need for the continuing review of commonly accepted screening procedures to assess their effectiveness. Although the distraction test has a place in the assessment of the hearing of children, its role has changed, and its function as a universal screening test is questionable.

We thank the staff of the hearing centre at the Royal Hospital for Sick Children, Bristol and Dr Paul Kinnersley for help with this study.

1 British Association of Audiological Physicians. Policy document on paediatric audiology. London: BAAP, 1989. Hall DMB, ed. Health for all children. A programme for child health surveillance. 2nd Ed. Oxford: Oxford University Press, 1991

3 McCormick B. Hearing screening by health visitors: a critical appraisal of the distraction test. Health Visito 1983; 56: 449-51.

4 Colver AF. Health surveillance of pre-school children: four years' experience. $B M F$ 1990; 300: 1246-8.

5 Peckham CS. Hearing impairment in childhood. $\mathrm{Br} \mathrm{Med}$ Bull 1986; 42: 145-9.

6 Watkin PM, Baldwin M, Laoide S. Parental suspicion and identification of hearing impairment. Arch Dis Child 1990 65: $846-50$. 
7 Boothman R, Orr N. Value of screening for deafness in the first year of life. Arch Dis Child 1978; 53: 570-3.

8 Brown J, Watson E, Alberman E. Screening infants for hearing loss. Arch Dis Child 1989; 64: 1488-95.

9 Johnson A, Ashurst H. Screening for sensorineural deafness by health visitors. Arch Dis Child 1990; 65: 841-5.

10 Haggard M, Hughes E. Screening children's hearing. London: HMSO, 1991 .

11 Research Group on the Effectiveness of Health Service. The treatment of persistent glue ear in children. Effective health care. Leeds: School of Public Health, University of Leeds, 1992.

12 Scanlon PE, Bamford JM. Early identification of hearing loss: screening and surveillance methods. Arch Dis Child 1990; 65: 479-85.

13 Wilson JMG, Junger G. Principles and practice of screening for disease. Geneva: WHO, 1968.

14 Sackett DL, Haynes RB, Tugwell P. Clinical epidemiology. A basic science for clinical medicine. Boston: Little, Brown, 1985. 